\title{
Brachial-ankle pulse wave velocity and metabolic syndrome in general population: the APAC study
}

\author{
Anxin Wang ${ }^{1,2,3,4,5+}$, Zhaoping Su ${ }^{6 \dagger}$, Xiaoxue Liư ${ }^{7}$, Yuling Yang ${ }^{8}$, Shuohua Chen ${ }^{9}$, Suzhen Wang ${ }^{6}$, Yanxia Luo ${ }^{5}$,
} Xiuhua Guo ${ }^{5^{*}}$, Xingquan Zhao ${ }^{1,2,3,4^{*}}$ and Shouling $\mathrm{Wu}^{9^{*}}$

\begin{abstract}
Background: Metabolic syndrome (MetS) is correlated with arterial stiffness and can be evaluated by brachialankle pulse wave velocity (baPWV). We investigated potential associations between MetS and baPWV in a Chinese community population.

Methods: The community-based Asymptomatic Polyvascular Abnormalities in Community study examined asymptomatic polyvascular abnormalities in a Chinese population aged $\geq 40$ years. The relationship between MetS and its components and baPWV was analyzed by multivariate logistic and linear regression models.

Results: Out of 5181 study participants, 1271 subjects (24.53\%) had MetS. Mean values of baPWV in subjects with $0,1,2,3,4$, and 5 components of MetS were 1430, 1526, 1647, 1676,1740, and $1860 \mathrm{~cm} / \mathrm{s}$, respectively $(p<0.001$ for trend). After adjusting for confounding risk factors, MetS was significantly associated with baPWV (odds ratio [OR]: 2.74; $95 \% \mathrm{Cl}: 2.28,3.30$ ). Among the five components of MetS, elevated blood pressure was the most important factor for baPWV. All models of multivariate linear regression analysis showed a significant positive correlation between the increasing numbers of MetS components and baPWV $(p<0.0001)$.
\end{abstract}

Conclusions: baPWV was associated with MetS and was greater with increasing numbers of MetS components. Elevated blood pressure was the most important factor for baPWV.

Keywords: Brachial-ankle pulse wave velocity, Metabolic syndrome, Arterial stiffnes, Cardio-cerebrovascular diseases

\section{Background}

Metabolic syndrome (MetS) is a pre-atherosclerotic state which involves a cluster of factors, such as abdominal obesity, arterial hypertension, dyslipidemia, and hyperglycemia, and is closely associated with a marked increase in the risk of cardio-cerebrovascular diseases and type 2

\footnotetext{
*Correspondence: statguo@ccmu.edu.cn; zxq@vip.163.com; drwusl@163.com ${ }^{\dagger}$ Equal contributors

${ }^{5}$ Department of Epidemiology and Health Statistics, School of Public Health, Capital Medical University, Beijing 100069, People's Republic of China 'Department of Neurology, Beijing Tiantan Hospital, Capital Medical University, No. 6 Tiantanxili, Dongcheng District, Beijing 100050, People's Republic of China

${ }^{9}$ Department of Cardiology, Kailuan Hospital, North China University of Science and Technology, No. 57 Xinhua Road, Lubei District, Tangshan 063000, People's Republic of China

Full list of author information is available at the end of the article
}

diabetes mellitus [1-3]. Some studies have shown that MetS is correlated with arterial stiffness. Nakanishi et al. suggested that persistent MetS conditions can deteriorate the severity of arterial stiffness [4, 5]. Furthermore, Tomiyama et al. suggested that an improvement in MetS status could delay the progression of atherosclerosis [6].

Pulse wave velocity is an objective valid index of arterial stiffness and is widely used for non-invasive evaluation of subclinical atherosclerotic changes [7]. Brachial-ankle pulse wave velocity (baPWV), a type of pulse wave velocity, is a promising test method that can assess the stiffness of both aortic and peripheral arteries [8,9]. baPWV not only reflects early atherosclerotic change [10-12], but also serves as a valuable predictor of mortality due to cardio-cerebrovascular events [13]. In the past decade, studies have investigated the relationship between arterial 
stiffness measured by baPWV and the components of MetS in different populations worldwide. However, the association of baPWV with MetS is not understood well. The correlation between baPWV and MetS and its components may be different based on different populations, regions and cultural diet.

The purpose of the present study was to evaluate the relationship between baPWV and MetS and its individual components in a large sample size of 5181 communitybased subjects in the northern region of China, and to further elucidate the complex correlation between baPWV and MetS.

\section{Methods}

\section{Study population}

The Asymptomatic Polyvascular Abnormalities Community (APAC) study is a community-based observational prospective, long-term follow-up study to investigate the epidemiology of asymptomatic polyvascular abnormalities in Chinese adults [14]. The study cohort was a subgroup of a previously described population of the Kailuan study [15]. Data about patient demographics, clinical characteristics (physical examination, laboratory tests, carotid duplex ultrasound and transcranial Doppler examinations), and also finished structured interviews with a standardized questionnaire performed by trained investigators. Anthropometric indices included height and weight. Body mass index (BMI) was calculated as body weight $(\mathrm{kg})$ divided by the square of body height $\left(\mathrm{m}^{2}\right)$. Blood pressure was measured twice with an appropriate cuff size in relation to arm size, in the participants with the supine position after $10 \mathrm{~min}$ resting. The average of the two readings was used. However, if the difference between the two measurements exceeded $5 \mathrm{mmHg}$, a third reading would be conducted, and the average of three readings was recorded and analyzed. Smoking was defined as at least one cigarette per day for more than a year. Alcohol abuse was defined as alcohol intake of at least 90 and $45 \mathrm{~g}$ of liquor a day for men and women, respectively, for more than 1 year. Smoking or drinking cessation was considered only if it lasted for at least 1 year. Details of the APAC study design and the information on baseline characteristics have been published previously. The community is part of a large coal mining industry in Tangshan, Hebei Province $[15,16]$, China. The study protocol was approved by the Ethics Committees of the Kailuan General Hospital and the Beijing Tiantan Hospital, and informed consent was obtained from all participants.

\section{Definition of MetS}

MetS was defined using previously published criteria from the International Diabetes Federation [11]. The definition included the presence of central obesity (waist circumference $>90 \mathrm{~cm}$ for Chinese men and $>80 \mathrm{~cm}$ for Chinese women), plus any two of the following factors: triglycerides $>150 \mathrm{mg} / \mathrm{dl}(1.7 \mathrm{mmol} / \mathrm{L})$ or obtaining therapy for increased triglyceride (TG) concentrations; highdensity lipoprotein cholesterol (HDL-C), $40 \mathrm{mg} / \mathrm{dl}$ (1.03 $\mathrm{mmol} / \mathrm{L})$ in men and $50 \mathrm{mg} / \mathrm{dl}(1.29 \mathrm{mmol} / \mathrm{L})$ in women, or obtaining therapy for low HDL-C level; systolic blood pressure $(\mathrm{BP})>130 \mathrm{mmHg}$ or diastolic $\mathrm{BP}>85 \mathrm{mmHg}$, or treatment of previously diagnosed arterial hypertension; fasting plasma glucose $>100 \mathrm{mg} / \mathrm{dl}(5.6 \mathrm{mmol} / \mathrm{L})$ or previously diagnosed type 2 diabetes mellitus.

\section{Measurement of baPWV}

Bilateral baPWV was evaluated by utilizing aBP-203RPE III device ( Omron Healthcare, Kyoto, Japan). Details about this instrument and its use have been described, and clinical validation and good reproducibility have been confirmed [8]. All examinations were carried out by specially trained physicians and nurses. All subjects were light clothing and underwent examination under resting-state by placement of the subject in a supine position without a pillow. Electrodes of the electrocardiograph were located in both wrists, a stethoscope was located in the left border of the sternum for acquiring heart sounds, and cuffs were wrapped with certain strain on both the upper arms and ankles. The lower border of the brachial cuff was located $2-3 \mathrm{~cm}$ above the chelidon, and the lower border of ankle cuff was located 1-2 cm above the medial malleolus. Two measured values were recorded from both sides of the body, while the second value of baPWV was utilized. Then we used the higher of the left and right baPWV values for analyses [17].

Arterial stiffness was considered as baPWV $\geq 1400 \mathrm{~cm} / \mathrm{s}$, since this is what has been identified as an independent risk factor in the Framingham score and can distinguish patients with atherosclerotic cardiovascular disease. Diabetes was considered as fasting plasma glucose concentration of $\geq 7.0 \mathrm{mmol} / \mathrm{L}$ or treatment for diabetes with drug or insulin. Hypertension was defined as systolic BP $>130$ $\mathrm{mmHg}$ or diastolic $\mathrm{BP}>85 \mathrm{mmHg}$ or on drug treatment for hypertension.

\section{Statistical analysis}

The continuous variables and categorical data are expressed as the mean \pm standard deviation (SD) and percentage, respectively. The Student's t-test or ANOVA test was used to address non-paired samples for the comparison of normally distributed parameters, and the Wilcoxon or Kruskal-Wallis test for the comparison of non-parametric variables. The qualitative variables were compared by using chi-squared test. Four multivariate logistic regression analyses were performed to calculate odds ratio (OR) and 95\% confidence interval (CI) for the associations of MetS or the number of MetS components (the 0 MetS component 
group was used as the reference category). Arterial stiffness was defined as baPWV $\geq 1400 \mathrm{~cm} / \mathrm{s}$. Model 1 was the crude model; Model 2 adjusted for age and gender; Model 3 adjusted for age, gender, level of education, income, smoking, alcohol abuse, and physical activity; and Model 4 adjusted for age, gender, level of education, income, smoking, alcohol abuse, amount of physical activity, BMI, and serum concentrations of low-density lipoprotein cholesterol
(LDL-C) and high-sensitivity C-reactive protein. Finally, for each model, a trend test was performed after the number of MetS components as a continuous variable entering into the model. Furthermore, a multivariate linear regression was applied to analyze the independent associations between baPWV and MetS. Differences were considered statistically significant only when $p$-value was $<0.05$. All data were analyzed with a commercially available software

Table 1 Clinical and biochemical characteristics of subjects with and without metabolic syndrome

\begin{tabular}{|c|c|c|c|c|}
\hline Variables & Total $(n=5181)$ & MetS group $(n=1271)$ & Non-MetS group $(n=3910)$ & $P$ value \\
\hline Age (year) & $55.16 \pm 11.80$ & $56.29 \pm 10.47$ & $54.79 \pm 12.18$ & $<0.0001$ \\
\hline Male, n (\%) & $3108(59.99)$ & $701(55.15)$ & $2407(61.56)$ & $<0.0001$ \\
\hline \multicolumn{5}{|l|}{ Education, n (\%) } \\
\hline Illiteracy/Primary School & $638(12.31)$ & $188(14.79)$ & $450(11.51)$ & \multirow[t]{3}{*}{0.0004} \\
\hline Middle School & $2295(44.30)$ & $583(45.87)$ & $1712(43.79)$ & \\
\hline High School or Higher & $2248(43.39)$ & $500(39.34)$ & $1748(44.71)$ & \\
\hline \multicolumn{5}{|l|}{ Income, n (\%) } \\
\hline$<¥ 1000$ & $1239(23.91)$ & $264(20.77)$ & $975(24.94)$ & \multirow[t]{3}{*}{0.0001} \\
\hline$¥ 1000-3000$ & $3431(66.22)$ & $903(71.05)$ & $2528(64.65)$ & \\
\hline$>¥ 3000$ & $511(9.86)$ & $104(8.18)$ & $407(10.41)$ & \\
\hline Body Mass Index (kg/m2) & $24.94 \pm 3.25$ & $27.38 \pm 3.00$ & $24.15 \pm 2.92$ & $<0.0001$ \\
\hline Waist circumference (cm) & $86.12 \pm 9.66$ & $93.90 \pm 7.26$ & $83.59 \pm 8.96$ & $<0.0001$ \\
\hline Waist-to hip ratio & $0.88 \pm 0.06$ & $0.91 \pm 0.06$ & $0.87 \pm 0.06$ & $<0.0001$ \\
\hline Systolic Blood Pressure (mmHg) & $131.33 \pm 20.04$ & $141.18 \pm 18.36$ & $128.12 \pm 19.51$ & $<0.0001$ \\
\hline Diastolic Blood Pressure (mmHg) & $82.86 \pm 11.06$ & $88.10 \pm 10.79$ & $81.16 \pm 10.60$ & $<0.0001$ \\
\hline Fasting Plasma Glucose (mmol/L) & $5.59 \pm 1.51$ & $6.37 \pm 2.01$ & $5.33 \pm 1.20$ & $<0.0001$ \\
\hline Total cholesterol (mmol/L) & $1.53 \pm 0.70$ & $1.69 \pm 0.74$ & $1.48 \pm 0.68$ & $<0.0001$ \\
\hline Triglycerides (mmol/L) & $1.68 \pm 1.42$ & $2.60 \pm 2.01$ & $0.38 \pm 1.00$ & $<0.0001$ \\
\hline High-Density Lipoprotein-Cholesterol ( mmol/L) & $1.63 \pm 0.46$ & $1.45 \pm 0.41$ & $1.68 \pm 0.46$ & $<0.0001$ \\
\hline Low-Density Lipoprotein-Cholesterol (mmol/L) & $2.63 \pm 0.74$ & $2.76 \pm 0.87$ & $2.58 \pm 0.69$ & $<0.0001$ \\
\hline High-Sensitivity C-Reactive Protein & $2.13 \pm 4.18$ & $2.77 \pm 4.25$ & $1.92 \pm 4.14$ & $<0.0001$ \\
\hline bapwv (cm/s) & $1588.40 \pm 401.62$ & $1703.30 \pm 371.57$ & $1551.06 \pm 403.98$ & $<0.0001$ \\
\hline Hypertension, n (\%) & $2500(48.25)$ & $980(77.10)$ & $1520(38.87)$ & $<0.0001$ \\
\hline Diabetes mellitus, n (\%) & $629(12.14)$ & $348(27.38)$ & $281(7.19)$ & $<0.0001$ \\
\hline \multicolumn{5}{|l|}{ Smoking, n (\%) } \\
\hline Never & $3233(62.40)$ & $812(63.89)$ & $2421(61.92)$ & \multirow[t]{3}{*}{0.3655} \\
\hline Former & $291(5.62)$ & $73(5.74)$ & $218(5.58)$ & \\
\hline Current & 1657 (31.98) & $386(30.37)$ & $1271(32.51)$ & \\
\hline \multicolumn{5}{|l|}{ Alcohol abuse, n (\%) } \\
\hline Never & 3391 (65.51) & $833(65.54)$ & $2561(65.50)$ & \multirow[t]{3}{*}{0.7074} \\
\hline Former & $81(1.56)$ & $23(1.81)$ & $58(1.48)$ & \\
\hline Current & 1706 (32.93) & 415 (32.65) & $1291(33.02)$ & \\
\hline \multicolumn{5}{|l|}{ Physical Activity, n (\%) } \\
\hline Inactive & $2087(40.28)$ & $506(39.81)$ & $1581(40.43)$ & \multirow[t]{3}{*}{0.6289} \\
\hline Moderately Active & $1312(25.32)$ & $314(24.70)$ & $998(25.52)$ & \\
\hline Very Active & $1782(34.39)$ & $451(35.48)$ & $1331(34.04)$ & \\
\hline
\end{tabular}

MetS metabolic syndrome, baPWV brachial-ankle pulse wave velocity 
program (SAS software version 9.3; SAS Institute Inc., Cary, NC, USA).

\section{Results}

Of the 5440 subjects who were originally included into the APAC study, we excluded 218 participants without measurement of baPWV and 41 individuals without MetS. Eventually, 5181 participants (3108 men, 2073 women) with a mean age of $55.16 \pm 11.80$ years (range: 40-94 years) were included in the present investigation. The distribution of demographics, anthropometric measurement, baPWV, vascular risk factors and living habits according to presence of metabolic syndrome are summarized in Table 1. At baseline, 1271 out of 5181 subjects $(24.53 \%)$ had MetS. Compared with those with no MetS, the incidence of MetS was found significantly in older subjects, in higher proportion of men, in subjects with lower level of education, subjects at the middleincome level $(p<0.001$ for all). There were no statistically significant differences for smoking $(p=0.3655)$, drinking $(p=0.7074)$ and physical activity $(p=0.6289)$ between the MetS group and the group without MetS. The baPWV values were significantly and positively correlated with the values of the components of MetS $(p<0.0001)$.

Mean values of baPWV in subjects with $0,1,2,3,4$, and 5 features of MetS were 1430, 1526, 1647, 1676,1740 , and $1860 \mathrm{~cm} / \mathrm{s}$, respectively $(p<0.001$ for trend) (Fig. 1).
The characteristics of the participants in relation to baPWV are shown in Table 2. There were significant differences between the two groups in mean age and sex $(p<0.0001$ for both). The percentage of MetS and its components was higher in the arterial stiffness group (baPWV $\geq 1400 \mathrm{~cm} / \mathrm{s}$ ) than the non-arterial stiffness group (baPWV $<1400 \mathrm{~cm} / \mathrm{s}$ ), and a higher proportion of subjects in the arterial stiffness group than those in the non-arterial stiffness group had exceeded thresholds for BMI, waist circumference, waist-to-hip ratio, systolic BP, diastolic BP, fasting plasma glucose, total cholesterol, triglycerides, HDL-C, LDL-C, and high-sensitivity Creactive protein $(p<0.0001$ for all). There was a trend toward higher age, education, hypertension, diabetes mellitus, smoking, alcohol abuse, and less or no physical activity in the arterial stiffness group than in the nonarterial stiffness group $(p<0.0001$ for all). The severity of central obesity, elevated arterial BP, raised fasting plasma glucose and TG, and reduced HDL-C concentration and the degree of MetS were significantly higher in the arterial stiffness group than in the group without arterial stiffness.

In all four models of the multivariable logistic regression analysis, the risk of arterial stiffness increased significantly $(p<0.0001)$ with increasing number of MetS components (Table 3). After adjustment for age, gender, level of education, income, smoking, alcohol abuse, amount of physical activity, BMI, and serum concentrations of LDL-C, high-sensitivity C-reactive protein (Model 4), MetS remained significantly associated with

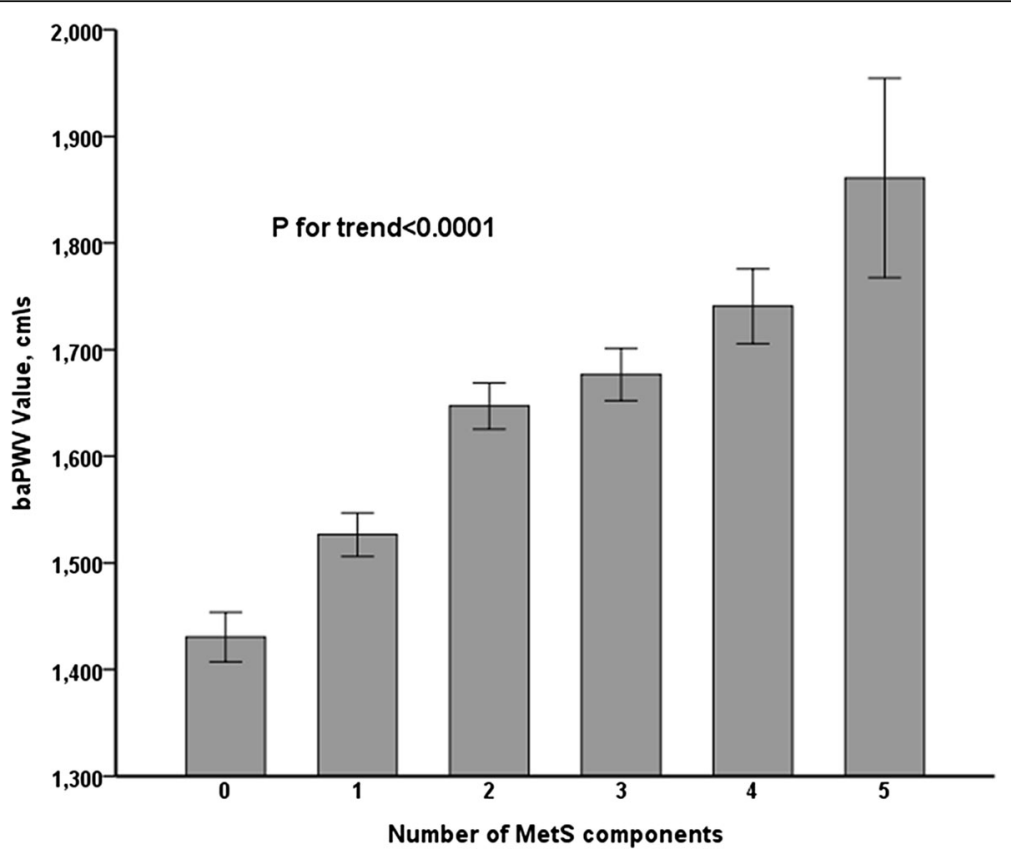

Fig. 1 Mean baPWV according to the number $(0,1,2,3,4,5)$ of components of metabolic syndrome. MetS: metabolic syndrome; baPWV: brachial ankle pulse wave velocity 
Table 2 Clinical and biochemical characteristics of baPWV

\begin{tabular}{|c|c|c|c|}
\hline Variables & $\mathrm{baPWV}<1400 \mathrm{~cm} / \mathrm{s}(n=1965)$ & $\mathrm{baPWV} \geq 1400 \mathrm{~cm} / \mathrm{s}(n=3216)$ & $P$ value \\
\hline Age (year) & $48.14 \pm 6.39$ & $59.45 \pm 12.28$ & $<0.0001$ \\
\hline Male, $n$ & $944(48.04)$ & $2164(67.29)$ & $<0.0001$ \\
\hline \multicolumn{4}{|l|}{ Education, n (\%) } \\
\hline Illiteracy/Primary School & $97(4.94)$ & $541(16.82)$ & \multirow[t]{3}{*}{$<0.000$} \\
\hline Middle School & $856(43.56)$ & $1439(44.75)$ & \\
\hline High School or Higher & $1012(51.50)$ & $1236(38.43)$ & \\
\hline \multicolumn{4}{|l|}{ Income, n (\%) } \\
\hline$<¥ 1000$ & $530(26.97)$ & $709(22.05)$ & \multirow[t]{3}{*}{$<0.0001$} \\
\hline$¥ 1000-3000$ & $1278(65.04)$ & $2153(66.95)$ & \\
\hline$>¥ 3000$ & $157(7.99)$ & $354(11.01)$ & \\
\hline Body Mass Index (kg/m2) & $24.05 \pm 3.09$ & $25.12 \pm 3.34$ & $<0.0001$ \\
\hline Waist circumference $(\mathrm{cm})$ & $83.36 \pm 9.55$ & $87.80 \pm 9.33$ & $<0.0001$ \\
\hline Waist-to hip ratio & $0.86 \pm 0.06$ & $0.89 \pm 0.06$ & $<0.0001$ \\
\hline Systolic Blood Pressure $(\mathrm{mmHg})$ & $118.78 \pm 14.16$ & $138.99 \pm 19.22$ & $<0.0001$ \\
\hline Diastolic Blood Pressure (mmHg) & $79.11 \pm 9.62$ & $85.15 \pm 11.25$ & $<0.0001$ \\
\hline Fasting Plasma Glucose (mmol/L) & $5.24 \pm 0.96$ & $5.80 \pm 1.73$ & $<0.0001$ \\
\hline Total cholesterol (mmol/L) & $1.42 \pm 0.64$ & $1.60 \pm 0.73$ & $<0.0001$ \\
\hline Triglycerides (mmol/L) & $1.50 \pm 1.25$ & $1.78 \pm 1.50$ & $<0.0001$ \\
\hline High-Density Lipoprotein-Cholesterol (mmol/L) & $1.67 \pm 0.47$ & $1.60 \pm 0.44$ & $<0.0001$ \\
\hline Low-Density Lipoprotein-Cholesterol (mmol/L) & $2.54 \pm 0.66$ & $2.68 \pm 0.78$ & $<0.0001$ \\
\hline High-Sensitivity C-Reactive Protein & $1.51 \pm 2.83$ & $2.51 \pm 4.79$ & $<0.0001$ \\
\hline Hypertension, n (\%) & $427(21.73)$ & $2073(64.46)$ & $<0.0001$ \\
\hline Diabetes mellitus, n (\%) & $94(4.78)$ & $535(16.64)$ & $<0.0001$ \\
\hline \multicolumn{4}{|l|}{ Smoking, n (\%) } \\
\hline Never & $1334(67.89)$ & $1899(59.05)$ & \multirow[t]{3}{*}{$<0.0001$} \\
\hline Former & $59(3.00)$ & $232(7.21)$ & \\
\hline Current & $572(29.11)$ & $1085(33.74)$ & \\
\hline \multicolumn{4}{|l|}{ Alcohol abuse, n (\%) } \\
\hline Never & $1401(71.30)$ & $1993(61.97)$ & \multirow[t]{3}{*}{$<0.0001$} \\
\hline Former & $18(0.92)$ & $63(1.96)$ & \\
\hline Current & $546(27.79)$ & $1160(36.07)$ & \\
\hline \multicolumn{4}{|l|}{ Physical Activity, n (\%) } \\
\hline Inactive & $877(44.63)$ & $1210(37.62)$ & \multirow[t]{3}{*}{$<0.0001$} \\
\hline Moderately Active & $561(28.55)$ & $751(23.35)$ & \\
\hline Very Active & $527(26.82)$ & $1255(39.02)$ & \\
\hline Metabolic Syndrome, n (\%) & $261(13.28)$ & $1010(31.41)$ & $<0.0001$ \\
\hline \multicolumn{4}{|l|}{ Metabolic Syndrome Components } \\
\hline Central Obesity, n (\%) & $907(46.16)$ & $1838(57.15)$ & $<0.0001$ \\
\hline Raised Triglycerides, n (\%) & $523(26.62)$ & $1152(35.82)$ & $<0.0001$ \\
\hline Reduced High-Density Lipoprotein-Cholesterol, n (\%) & $169(8.60)$ & 419 (13.03) & $<0.0001$ \\
\hline Raised Blood Pressure, n (\%) & $378(19.24)$ & $1820(56.59)$ & $<0.0001$ \\
\hline Raised Fasting Plasma Glucose, n (\%) & $455(23.16)$ & $1267(39.40)$ & $<0.0001$ \\
\hline \multicolumn{4}{|l|}{ Metabolic Syndrome (No. of Components) } \\
\hline $0, \mathrm{n}(\%)$ & $608(30.94)$ & $394(12.25)$ & $<0.0001$ \\
\hline
\end{tabular}


Table 2 Clinical and biochemical characteristics of baPWV (Continued)

\begin{tabular}{lll}
\hline $1, n(\%)$ & $644(32.77)$ & $751(23.35)$ \\
$2, n(\%)$ & $427(21.73)$ & $959(29.82)$ \\
$3, n(\%)$ & $216(10.99)$ & $681(21.18)$ \\
$4, n(\%)$ & $64(3.26)$ & $371(11.54)$ \\
$5, n(\%)$ & $6(0.31)$ & $60(1.87)$ \\
\hline
\end{tabular}

baPWV brachial ankle pulse wave velocity

baPWV (OR: 2.74; 95\% CI: 2.28-3.30). Among the five components of MetS, raised BP was the most important factor for baPWV.

The OR values were 4.60 for raised BP, 1.50 for raised fasting plasma glucose, 1.48 for reduced HDL-C, 1.38 for raised triglycerides, and 1.18 for central obesity. Using the subgroup with no component of MetS as baseline, the ORs for the associations between the subgroups with 1, 2, 3, 4 and 5 MetS components and baPWV were 1.97 (95\% CI: 1.60, 2.43), 3.80 (95\% CI: 3.04, 4.75), 5.70 (95\% CI: 4.38, 7.42), 11.66 (95\% CI: 8.16, 16.67), and 20.88 (95\% CI: 8.37, 52.04), respectively. In this multivariate model, the baPWV increased significantly ( $p<0.0001$ for trend) with the number of MetS components. The same held true for the three other models of the multivariate analysis (Table 3 ).

Figure 2 shows the results of multiple logistic regression analysis of the association between MetS components and baPWV in males and females (Model 4). In this model, metabolic syndrome and its components were associated with increased baPWV in both males and females. Among the five components of MetS, raised BP was still the most important factor for baPWV. Furthermore, regardless of gender, all models of the multivariate linear regression analysis in Table 4 showed a significant positive correlation between baPWV and MetS $(p<0.0001)$.

\section{Discussion}

In our investigation based on a community population in northern China, baPWV was significantly associated with MetS and baPWV, and there was greater statistical significance with increasing numbers of MetS components. Among the conditions of central obesity, raised triglycerides, reduced HDL-C, raised $\mathrm{BP}$, and raised fasting plasma glucose; raised BP was the strongest determinant for baPWV. Furthermore, the mean velocity of baPWV in subjects with five components of MetS had a 1.3 times faster than individuals with no MetS.

Table 3 Odds ratio and 95\% Cl of baPWV for MetS/components in multiple logistic regression analysis

\begin{tabular}{lllll}
\hline Variables & Model 1 & Model 2 & Model 3 & Model 4 \\
\hline Metabolic Syndrome & $2.99(2.57-3.47)$ & $2.99(2.54-3.53)$ & $2.93(2.48-3.46)$ & $2.74(2.28-3.30)$ \\
Central Obesity & $1.10(0.97-1.25)$ & $1.11(0.96-1.29)$ & $1.08(0.93-1.26)$ & $1.18(0.98-1.42)$ \\
Raised Triglycerides & $1.12(0.98-1.29)$ & $1.36(1.16-1.59)$ & $1.36(1.17-1.60)$ & $1.38(1.17-1.62)$ \\
Reduced High-Density Lipoprotein Cholesterol & $1.46(1.19-1.79)$ & $1.39(1.10-1.76)$ & $1.40(1.10-1.78)$ & $1.48(1.17-1.89)$ \\
Raised Blood Pressure & $4.99(4.36-5.71)$ & $4.51(3.88-5.25)$ & $4.44(3.82-5.18)$ & $4.60(3.93-5.39)$ \\
Raised Fasting Plasma Glucose & $1.76(1.54-2.01)$ & $1.60(1.38-1.14)$ & $1.58(1.35-1.84)$ & $1.50(1.28-1.76)$ \\
No. of Components & & & & \\
$\quad 0$ & Reference & Reference & Reference & Reference \\
$\quad 1$ & $1.80(1.53-2.12)$ & $1.89(1.55-2.31)$ & $1.87(1.53-2.29)$ & $1.97(1.60-2.43)$ \\
2 & $3.47(2.92-4.11)$ & $3.51(2.86-4.30)$ & $3.38(2.76-4.15)$ & $3.80(3.04-4.75)$ \\
3 & $4.86(3.99-5.94)$ & $4.98(3.96-6.27)$ & $4.80(3.81-6.05)$ & $5.70(4.38-7.42)$ \\
$\quad 4$ & $8.94(6.67-12.00)$ & $9.82(7.11-13.56)$ & $9.39(6.79-12.98)$ & $11.66(8.16-16.67)$ \\
5 & $15.43(6.60-36.06)$ & $15.80(6.51-38.32)$ & $15.35(6.30-37.38)$ & $20.88(8.37-52.04)$ \\
P for trend & $<0.0001$ & $<0.0001$ & $<0.0001$ & $<0.0001$
\end{tabular}

MetS metabolic syndrome, baPWV brachial ankle pulse wave velocity

Model 1: unadjusted

Model 2: adjusted for age and gender

Model 3: adjusted for age, gender, level of education, income, smoking, alcohol abuse, and amount of physical activity

Model 4: adjusted for age, gender, level of education, income, smoking, alcohol abuse, amount of physical activity, body mass index, and serum concentrations of low-density lipoprotein cholesterol, high-sensitivity C-reactive protein 


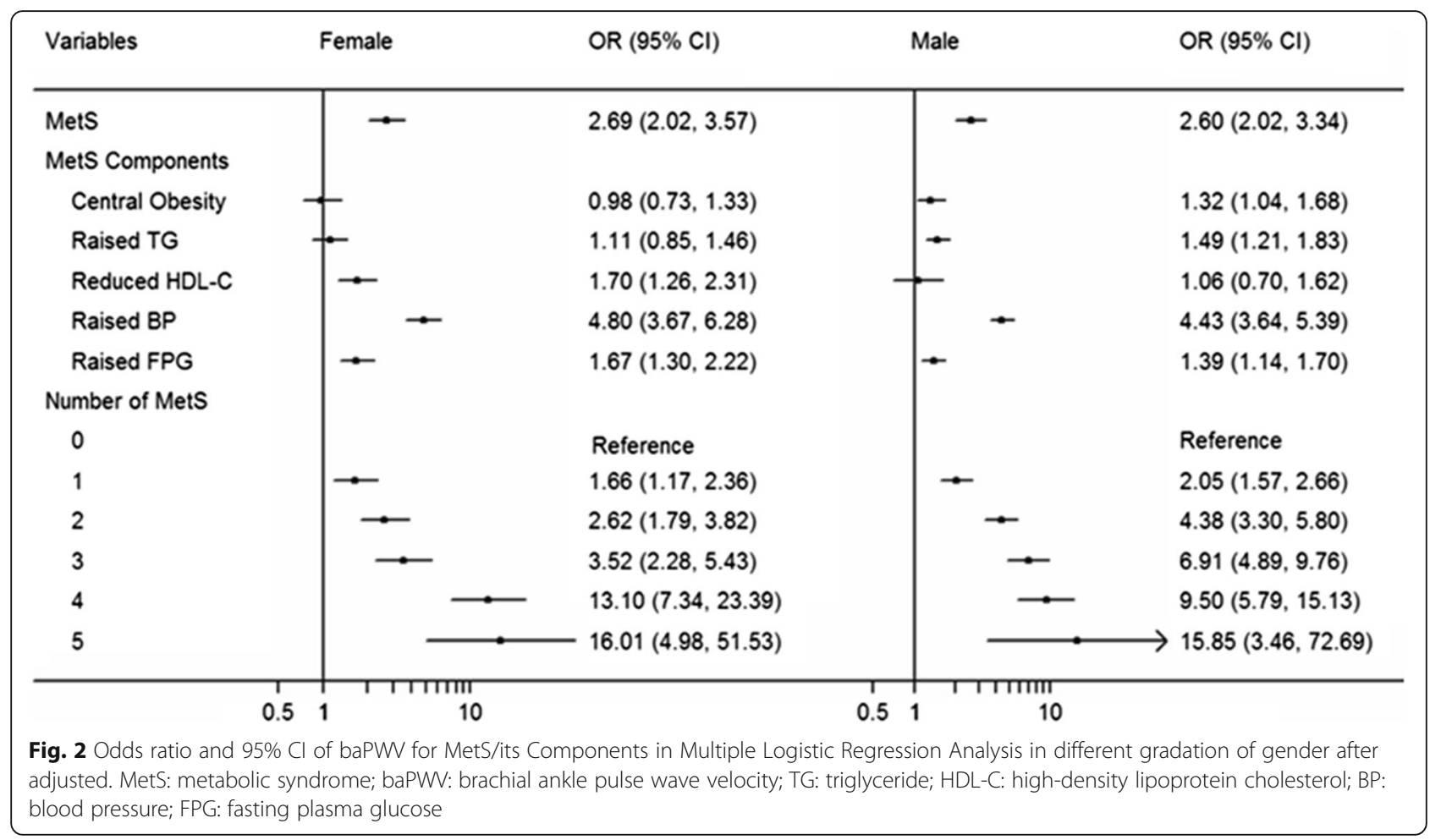

Table 4 Multivariate linear regression analysis models of associations between number of MetS components and the level of baPWV

\begin{tabular}{|c|c|c|c|c|c|}
\hline \multirow[t]{2}{*}{ Variables } & & \multicolumn{2}{|l|}{ MetS } & \multicolumn{2}{|c|}{ Increasing 1 component of MetS } \\
\hline & & $\mathrm{B}(95 \% \mathrm{Cl})$ & $\overline{P \text { Value }}$ & $\mathrm{B}(95 \% \mathrm{Cl})$ & $P$ Value \\
\hline & Model 1 & $152.24(127.16-177.32)$ & $<0.0001$ & $81.21(72.82-89.60)$ & $<0.0001$ \\
\hline & Model 2 & $125.39(106.03-144.75)$ & $<0.0001$ & $64.40(57.92-70.88)$ & $<0.0001$ \\
\hline & Model 3 & $122.17(102.80-141.53)$ & $<0.0001$ & $63.07(56.57-69.58)$ & $<0.0001$ \\
\hline & Model 4 & $132.74(111.13-154.35)$ & $<0.0001$ & $77.71(70.12-85.31)$ & $<0.0001$ \\
\hline \multicolumn{6}{|l|}{ Female } \\
\hline & Model 1 & $267.94(235.39-300.50)$ & $<0.0001$ & $118.25(107.83-128.66)$ & $<0.0001$ \\
\hline & Model 2 & $129.63(104.40-154.86)$ & $<0.0001$ & $59.27(50.69-67.85)$ & $<0.0001$ \\
\hline & Model 3 & $125.63(100.30-150.95)$ & $<0.0001$ & $58.03(49.38-66.68)$ & $<0.0001$ \\
\hline & Model 4 & $127.85(100.20-155.50)$ & $<0.0001$ & $66.78(56.80-76.76)$ & $<0.0001$ \\
\hline \multicolumn{6}{|l|}{ Male } \\
\hline & Model 1 & $82.90(47.96-117.83)$ & $<0.0001$ & $52.95(41.01-64.89)$ & $<0.0001$ \\
\hline & Model 2 & $107.28(79.16-135.40)$ & $<0.0001$ & $63.80(54.27-73.32)$ & $<0.0001$ \\
\hline & Model 3 & $105.18(77.09-133.26)$ & $<0.0001$ & $62.51(52.97-72.05)$ & $<0.0001$ \\
\hline & Model 4 & $126.18(94.48-157.89)$ & $<0.0001$ & 81.93 (70.80-93.06) & $<0.0001$ \\
\hline
\end{tabular}

MetS metabolic syndrome, baPWV brachial ankle pulse wave velocity

Model 1: unadjusted

Model 2: adjusted for age and gender

Model 3: adjusted for age, gender, level of education, income, smoking, alcohol abuse, and amount of physical activity

Model 4: adjusted for age, gender, level of education, income, smoking, alcohol abuse, amount of physical activity, body mass index, and serum concentrations of low-density lipoprotein cholesterol, high-sensitivity C-reactive protein 
Our findings demonstrate that components of MetS for the influence of vasculature appear to be additive. This result was consistent with those of other studies $[5,10,18]$. The individuals with more risk factors have substantially greater baPWV values than those with fewer risk factors, which can be explained by baPWV being closely associated with severity of arterial stiffness.

In a Japanese population study, Nakanishi et al. [5] showed that age-adjusted mean values of baPWV were increased with clustered features of MetS in both sexes. In addition, insulin resistance was closely associated with the risk for increased baPWV or increased arterial stiffness. However, in our study, raised BP was the strongest determinant for risk of increased baPWV. However, the mechanism by which MetS might increase baPWV remains unclear. One probable explanation for our findings is that elevated BP can increase baPWV by directly acting on the arterial wall.

Angelo Scuteri et al. [11] conducted a study involving nine cohorts representing eight different European countries and the United States and suggested that the "risky" cluster of raised triglycerides, raised BP, central obesity, raised fasting plasma glucose, and central obesity was significantly associated with extremely stiff arteries. They also showed the highest prevalence in Sardinian, Italian, British, and Belgian cohorts, and the lowest in cohorts from Sweden and the United States. However, Young-Kwon Kim [18] found that the other individual MetS components, except for a raised BP, do not affect arterial stiffness independently.

Another study [19] including 8599 subjects in south China suggested that baPWV was significantly higher in subjects with MetS than in those without MetS $(p<0.001$ for both sexes). All the metabolic components were correlated to baPWV in the male and female subjects except low HDL-C and high urine acid in the male group. BP and fasting plasma glucose had the strongest correlation factors. The baPWV values were positively correlated with advanced age $(p<0.001)$ and the values of the MetS components, and this correlation was stronger in females than in males $(p<0.001)$, which is different from the results summarized by Wang et al. [20].

Although the current study focused on the feasibility of a non-invasive baPWV examination as an effective tool to screen community populations at high risk of MetS, appropriate cut-off points or valid intervals of baPWV were not achieved [20-23]. With the marked growth of living standards in China, prevalence of MetS has increased and has resulted in serious social burden $[24,25]$. Arterial stiffness is an indicator of arterial damage and its increase with age has been demonstrated to be associated with an increased risk of cardio-cerebrovascular diseases [26-29]. The clinical implication of our study is that community populations with baPWV $>1400 \mathrm{~cm} /$ $\mathrm{s}$, advanced age, and MetS with reduced HDL-C, raised $\mathrm{BP}$ and raised fasting plasma glucose, might benefit from immediate intervention strategies which reduce the risk of further cardiovascular disease morbidity and mortality [30].

There are some potential limitations in our study. First, our community-based investigation, which is a cross-sectional investigation, cannot prove a causal relationship, since this has to be demonstrated in a longitudinal study. The data from this study only allows us to summarize the relationships between baPWV and MetS components, while the influence of MetS as a risk factor for cardio-cerebrovascular diseases may be shown in follow-up studies. Second, this study was based on the participants of the large Kailuan Study which included employees, retirees and their families of the Kailuan Company. Although we used a rigid random sampling method to reduce selection bias, the population of the Kailuan Company does not necessarily represent the populations of Hebei province. Finally, a crucial topic is the alteration of the results of the baPWV measurements by BP levels themselves.

\section{Conclusions}

In conclusion, our study is based on more than 5000 subjects, showing that increasing the number of MetS components is significantly correlated with a risk for increased baPWV in a northern China communitybased population. A decrease in arterial compliance or an increase in arterial stiffness was shown to be related to a risk for cardiovascular mortality, for this reason, individuals with clustered components of MetS might benefit from immediate interventions, including frequent exercise, reasonable diet regulation, and medical therapy for MetS. Furthermore, baPWV may be taken into consideration as a screening index in community-based healthcare plans and as a tool for evaluating the risk of cardiocerebrovascular diseases in clinical practice.

\section{Abbreviations \\ APAC: Asymptomatic polyvascular abnormalities community; baPW: brachial-ankle pulse wave velocity; BMI: Body mass index; BP: Blood pressure; Cl: Confidence internals; HDL-C: High-density lipoprotein cholesterol; LDL-C: Low-density lipoprotein cholesterol; MetS: Metabolic syndrome; OR: Odds ratio; SD: Standard deviation; TG: Triglyceride}

\section{Acknowledgements}

We thank all participating hospitals, colleagues, nurses, and imaging and laboratory technicians, as well as to the project development and management teams in Beijing Tiantan hospital and the Kailuan Group.

\section{Funding}

This study was supported by the Program of National Natural Science Foundation of China (Serial Number: 81530087 and 81473071), Natural Science Foundation of Shandong Province (ZR2013HM045) and the National Bureau of Statistics of China (2013LY013). 


\section{Availability of data and materials}

The datasets used and/or analysed during the current study available from the corresponding author on reasonable request.

\section{Authors' contributions}

$X Z$ and SW planned and designed the study. XL, YY, SC, SW, YL, XG contributed to the acquisition and interpretation of data. AW and ZS analyzed the data. AW and ZS were primarily responsible for writing the paper. XG, SW and XZ revised the manuscript for important intellectual content. All authors read and approved the final manuscript.

\section{Competing interests}

The authors declare that they have no competing interests.

\section{Consent for publication}

Not applicable.

\section{Ethics approval and consent to participate}

The study was approved by the Ethics Committees of both Kailuan General Hospital and Beijing Tiantan Hospital, and was in compliance with the Declaration of Helsinki. Written informed consent was obtained from all participants.

\section{Author details}

${ }^{1}$ Department of Neurology, Beijing Tiantan Hospital, Capital Medical University, No. 6 Tiantanxili, Dongcheng District, Beijing 100050, People's Republic of China. ${ }^{2}$ China National Clinical Research Center for Neurologica Diseases, Beijing, People's Republic of China. ${ }^{3}$ Center of Stroke, Beijing Institute for Brain Disorders, Beijing, People's Republic of China. ${ }^{4}$ Beijing Key Laboratory of Translational Medicine for Cerebrovascular Disease, Beijing, People's Republic of China. ${ }^{5}$ Department of Epidemiology and Health Statistics, School of Public Health, Capital Medical University, Beijing 100069 People's Republic of China. 'Department of Epidemiology and Health Statistics, Academy of public health and management, Weifang Medical University, No. 7166 Baotongxijie, Weicheng District, Weifang 261053 , People's Republic of China. 'Department of Cardiology, Tangshan People's Hospital, Tangshan, People's Republic of China. ${ }^{8} \mathrm{G}$ raduate School, North China University of Science and Technology, Tangshan, People's Republic of China. ${ }^{9}$ Department of Cardiology, Kailuan Hospital, North China University of Science and Technology, No. 57 Xinhua Road, Lubei District, Tangshan 063000, People's Republic of China.

\section{Received: 11 March 2016 Accepted: 14 November 2016}

Published online: 18 November 2016

\section{References}

1. Zhou F, Zhang H, Yao W, Mei H, Xu D, Sheng Y, Yang R, Kong X, Wang L, Zou J, et al. Relationship between brachial-ankle pulse wave velocity and metabolic syndrome components in a Chinese population. J Biomed Res. 2014;28(4):262-8

2. Alberti KG, Eckel RH, Grundy SM, Zimmet PZ, Cleeman Jl, Donato KA, Fruchart JC, James WP, Loria CM, Smith Jr SC. Harmonizing the metabolic syndrome: a joint interim statement of the International Diabetes Federation Task Force on Epidemiology and Prevention; National Heart, Lung, and Blood Institute; American Heart Association; World Heart Federation; International Atherosclerosis Society; and International Association for the Study of Obesity. Circulation. 2009;120(16):1640-5.

3. Alberti KG, Zimmet P, Shaw J. Metabolic syndrome-a new world-wide definition. A Consensus Statement from the International Diabetes Federation. Diabet Med. 2006;23(5):469-80.

4. Nakanishi N, Suzuki K, Tatara K. Clustered features of the metabolic syndrome and the risk for increased aortic pulse wave velocity in middleaged Japanese men. Angiology. 2003:54(5):551-9.

5. Nakanishi N, Shiraishi T, Wada M. Brachial-ankle pulse wave velocity and metabolic syndrome in a Japanese population: the Minoh study. Hypertens Res. 2005;28(2):125-31

6. Tomiyama H, Hirayama Y, Hashimoto H, Yambe M, Yamada J, Koji Y, Motobe K, Shiina K, Yamamoto Y, Yamashinai A. The effects of changes in the metabolic syndrome detection status on arterial stiffening: a prospective study. Hypertens Res. 2006;29(9):673-8.
7. Sipila K, Koivistoinen T, Moilanen L, Nieminen T, Reunanen A, Jula A, Salomaa V, Kaaja R, Koobi T, Kukkonen-Harjula K, et al. Metabolic syndrome and arterial stiffness: the Health 2000 Survey. Metabolism. 2007;56(3):320-6.

8. Yamashina A, Tomiyama H, Takeda K, Tsuda H, Arai T, Hirose K, Koji Y, Hori S, Yamamoto Y. Validity, reproducibility, and clinical significance of noninvasive brachial-ankle pulse wave velocity measurement. Hypertens Res. 2002;25(3):359-64.

9. Tomiyama H, Yamashina A, Arai T, Hirose K, Koji Y, Chikamori T, Hori S, Yamamoto Y, Doba N, Hinohara S. Influences of age and gender on results of noninvasive brachial-ankle pulse wave velocity measurement-a survey of 12517 subjects. Atherosclerosis. 2003;166(2):303-9.

10. Weng C, Yuan H, Tang X, Huang Z, Yang K, Chen W, Yang P, Chen Z, Chen $F$. Age- and gender dependent association between components of metabolic syndrome and subclinical arterial stiffness in a Chinese population. Int J Med Sci. 2012;9(8):730-7.

11. Scuteri A, Cunha PG, Rosei EA, Badariere J, Bekaert S, Cockcroft JR, Cotter J, Cucca F, De Buyzere ML, De Meyer T, et al. Arterial stiffness and influences of the metabolic syndrome: a cross-countries study. Atherosclerosis. 2014; 233(2):654-60.

12. Weng C, Yuan H, Yang K, Tang X, Huang Z, Huang L, Chen W, Chen F, Chen Z, Yang P. Gender-specific association between the metabolic syndrome and arterial stiffness in 8,300 subjects. Am J Med Sci. 2013;346(4):289-94.

13. Lehmann ED, Riley WA, Clarkson P, Gosling RG. Non-invasive assessment of cardiovascular disease in diabetes mellitus. Lancet. 1997;350 Suppl 1:SI14-9.

14. Zhou Y, Li Y, Xu L, Xu J, Wang A, Gao X, Wu S, Wei WB, Zhao X, Jonas JB. Asymptomatic polyvascular abnormalities in community (APAC) study in China: objectives, design and baseline characteristics. PLoS One. 2013;8(12):e84685.

15. Wang A, Liu X, Guo X, Dong Y, Wu Y, Huang Z, Xing A, Luo Y, Jonas JB, Wu $S$. Resting heart rate and risk of hypertension: results of the Kailuan cohort study. J Hypertens. 2014;32(8):1600-5. discussion 1605.

16. Wang A, Wu J, Zhou Y, Guo X, Luo Y, Wu S, Zhao X. Measures of adiposity and risk of stroke in China: a result from the Kailuan study. PLoS One. 2013; 8(4):e61665

17. Wang A, Tao J, Guo X, Liu X, Luo Y, Huang Z, Chen S, Zhao X, Jonas $J B, W u S$. The product of resting heart rate times blood pressure is associated with high brachial-ankle pulse wave velocity. PLoS One. 2014;9(9):e107852.

18. Kim YK. Impact of the metabolic syndrome and its components on pulse wave velocity. Korean J Intern Med. 2006;21(2):109-15.

19. Chen L, Zhu W, Mai L, Fang L, Ying K. The association of metabolic syndrome and its components with brachial-ankle pulse wave velocity in south China. Atherosclerosis. 2015;240(2):345-50

20. Wang G, Zheng L, Li X, Wu J, Zhang L, Zhang J, Zou L, Zhang Y, Zhou Q, Fan $\mathrm{H}$, et al. Using brachial-ankle pulse wave velocity to screen for metabolic syndrome in community populations. Sci Rep. 2015:5:9438.

21. Yamashina A, Tomiyama H, Arai T, Hirose K, Koji Y, Hirayama Y, Yamamoto $Y$, Hori S. Brachial-ankle pulse wave velocity as a marker of atherosclerotic vascular damage and cardiovascular risk. Hypertens Res. 2003;26(8):615-22.

22. Lin WY, Lai MM, Li Cl, Lin CC, Li TC, Chen CC, Lin T, Liu CS. In addition to insulin resistance and obesity, brachial-ankle pulse wave velocity is strongly associated with metabolic syndrome in Chinese-a population-based study (Taichung Community Health Study, TCHS). J Atheroscler Thromb. 2009; 16(2):105-12.

23. Kim YS, Kim DH, Choi BH, Sohn EH, Lee AY. Relationship between brachialankle pulse wave velocity and cognitive function in an elderly communitydwelling population with metabolic syndrome. Arch Gerontol Geriatr. 2009; 49(1):176-9.

24. Wu S, Lin H, Zhang C, Zhang Q, Zhang D, Zhang Y, Meng W, Zhu Z, Tang F, Xue $F$, et al. Association between erythrocyte parameters and metabolic syndrome in urban Han Chinese: a longitudinal cohort study. BMC Public Health. 2013;13:989.

25. Workalemahu T, Gelaye B, Berhane Y, Williams MA. Physical activity and metabolic syndrome among Ethiopian adults. Am J Hypertens. 2013;26(4):535-40.

26. Laurent S, Cockcroft J, Van Bortel L, Boutouyrie P, Giannattasio C, Hayoz D, Pannier B, Vlachopoulos C, Wilkinson I, Struijker-Boudier H. Expert consensus document on arterial stiffness: methodological issues and clinical applications. Eur Heart J. 2006;27(21):2588-605.

27. McEniery CM, Spratt M, Munnery M, Yarnell J, Lowe GD, Rumley A, Gallache J, Ben-Shlomo Y, Cockcroft JR, Wilkinson IB. An analysis of prospective risk factors for aortic stiffness in men: 20-year follow-up from the Caerphilly prospective study. Hypertension. 2010;56(1):36-43. 
28. Wang KL, Cheng HM, Sung SH, Chuang SY, Li CH, Spurgeon HA, Ting CT, Najjar SS, Lakatta EG, Yin FC, et al. Wave reflection and arterial stiffness in the prediction of 15-year all-cause and cardiovascular mortalities: a community-based study. Hypertension. 2010;55(3):799-805.

29. Vlachopoulos C, Aznaouridis K, Stefanadis C. Prediction of cardiovascular events and all-cause mortality with arterial stiffness: a systematic review and meta-analysis. J Am Coll Cardiol. 2010;55(13):1318-27.

30. Grundy SM, Cleeman Jl, Daniels SR, Donato KA, Eckel RH, Franklin BA, Gordon DJ, Krauss RM, Savage PJ, Smith Jr SC, et al. Diagnosis and management of the metabolic syndrome: an American Heart Association/ National Heart, Lung, and Blood Institute Scientific Statement. Circulation. 2005;112(17):2735-52.

Submit your next manuscript to BioMed Central and we will help you at every step:

- We accept pre-submission inquiries

- Our selector tool helps you to find the most relevant journal

- We provide round the clock customer support

- Convenient online submission

- Thorough peer review

- Inclusion in PubMed and all major indexing services

- Maximum visibility for your research

Submit your manuscript at www.biomedcentral.com/submit
Biomed Central 